Pacific Journal of Mathematics

FIXED POINTS FOR ORIENTATION PRESERVING
HOMEOMORPHISMS OF THE PLANE WHICH INTERCHANGE
TWO POINTS

Emilio Gagliardo AND CLIFFord ALFons Kottman 


\title{
FIXED POINTS FOR ORIENTATION PRESERVING HOMEOMORPHISMS OF THE PLANE WHICH INTERCHANGE TWO POINTS
}

\author{
Emilio Gagliardo and Clifford Kottman
}

Let $T$ be an orientation preserving homeomorphism defined on a subset of the plane which interchanges two points, $P$ and $Q$. Let $\Gamma$ be a simple curve joining $P$ and $Q$ and let $\Omega$ be a simply connected set contained in the domain and range of $T$ such that $\Gamma \subset \Omega, T(\Gamma) \subset \Omega, T^{-1}(\Gamma) \subset \Omega$. Then $T$ has a fixed point in $\Omega$. A corollary concerning fixed points of homeomorphisms on $S^{2}$ follows.

The proof would be trivial if $T$ were necessarily an element of a flow on the plane, however an example given in this paper shows that this need not be the case.

If, in particular, $T$ is defined in the whole plane or if its domain and range are the same halfplane, then the existence (but no constructive information about the location) of a fixed point could also be derived from classical results of Brouwer [2] (see for instance Proposition $0(a \Rightarrow b)$ and Proposition 1.1 of S. A. Andrea [1]).

1. The theorem. We use $\mathscr{R}$ to denote the real numbers and $\mathscr{R}^{2}$ for the coordinate plane. A curve is a continuous function whose domain is a compact interval of $\mathscr{R}$ and whose range is a subset of $\mathscr{R}^{2}$. If $[a, b]$ is an interval within the domain of the curve $\Phi$, we use $\Phi[a, b]$ as a shorthand for $\{\Phi(t): t \in[a, b]\} ; \Phi(a, b)$ and $\Phi[a, b)$ have analogous meanings. The terms close surve, simple curve, and simple closed curve have the standard meanings.

For the following lemmas we fix two simple closed curves, $\Phi_{1}$ and $\Phi_{2}:[0,3] \rightarrow \mathscr{R}^{2}$. The first, $\Phi_{1}$, is the triangle defined by

$$
\Phi_{1}(t)= \begin{cases}(2 t-1,2 t) & \text { for } 0 \leqq t \leqq 1 \\ (1,4-2 t) & \text { for } 1 \leqq t \leqq 2 \\ (5-2 t, 0) & \text { for } 2 \leqq t \leqq 3\end{cases}
$$

Referring to Figure $1, \Phi_{1}[0,1]$ is the segment $M H, \Phi_{1}[1,2]$ is the segment $H K$, and $\Phi_{1}[2,3]$ is the segment $K M$.

The second curve, $\Phi_{2}$, is defined so that the following conditions are satisfied: 
( I ) $\Phi_{2}[0,1]$ is the segment from $L=(\lambda, 0)$ (with $\lambda>0$ ) to $M=(-1,0)$; one has therefore: $\Phi_{2}(\rho)=0=(0,0)$ for a suitable $\rho$ with $0<\rho<1$.

(II) $\Phi_{2}[0,3]$ has winding number -1 about each of its interior points (just as $\Phi_{1}$ has); and therefore $\Phi_{2}[1,3]$ has winding number $-1 / 2$ about the origin (just as $\Phi_{1}[0,2]$ has).

(III) $\Phi_{2}(1,2]$ is disjoint from $\Phi_{1}[1,3]$. In Figure $1, \Phi_{2}[1,2]$ is represented by the curve $M N$, which except for $M$ is disjoint from $H K$ and $K M$.

(IV) $\Phi_{2}[2,3)$ is disjoint from $\Phi_{1}[2,3]$. In Figure $1, \Phi_{2}[2,3]$ is represented by the curve $N L$, which except perhaps for $L$, is disjoint from $K M$.

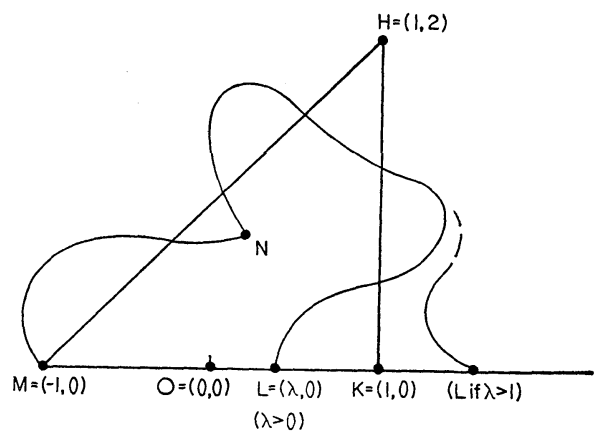

FIGURE 1

LEMMA 1. With $\Phi_{1}$ and $\Phi_{2}$ as above, the closed curve $\Psi(t)=$ $\Phi_{1}(t)-\Phi_{2}(t), 0 \leqq t \leqq 3$, has winding number -1 about the origin.

Proof. It is clear that $\Psi[0,3]$ is a closed curve with $\Psi(t) \neq(0,0)$ for all $t$, so that the winding number of $\Psi$ about the origin is defined. The idea of the following proof is to deform $\Psi$ without touching the origin, into a curve which obviously has winding number -1 about the origin.

Let

$$
\Xi(u, v)=\Phi_{1}(u)-\Phi_{2}(v) \text {. }
$$

From conditions (I) (II) (III) (IV) it follows rather easily that $\Xi^{-1}(0)$ is a subset of the hatched area in Figure 2. For our purpose it is enough to prove that the origin is never in the range of $\Xi(u, v)$ restricted to the dotted region in Figure 2, i.e. the region bounded by the segment $A G$ and the piecewise linear curve $\Sigma=A B C D E F G$ where $A=(0,0), B=(0, \rho), C=(2, \rho), D=(2,1), E=(5 / 2,1), F=(5 / 2$, $3), G=(3,3)$. In details: for $0 \leqq u \leqq 1,0 \leqq v \leqq \rho, \Phi_{1}(u)$ is a point of the segment $M H$ wnile $\Phi_{2}(v)$ is on the segment $L O$, hence $\Phi_{1}(u) \neq$ $\Phi_{2}(v)$; for $0<u \leqq 2, \rho \leqq v \leqq 1, \Phi_{1}(u)$ is on one of the segmehts $M H$, 


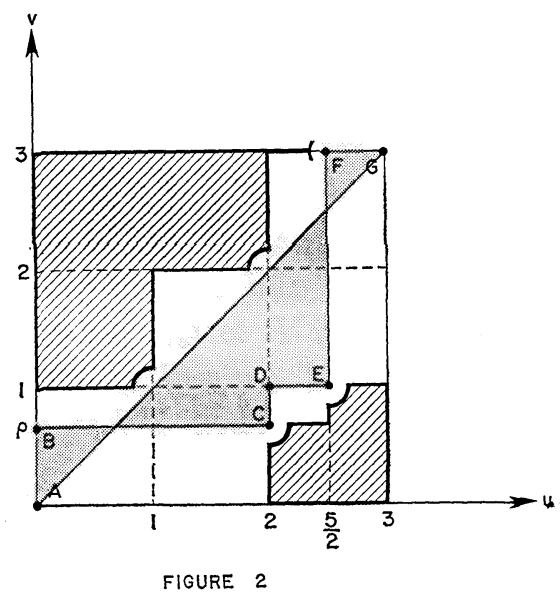

FIGURE 2

$H K$ but not at $M$ (since $u>0$ ) while $\Phi_{2}(v)$ is on the segment $O M$, hence again $\Phi_{1}(u) \neq \Phi_{2}(v)$; for $1 \leqq u \leqq 5 / 2,1 \leqq v \leqq 2, \Phi_{1}(u)$ is on one of the segments $H K, K O$ while $\Phi_{2}(v)$ is a point of the curve $M N$ which by condition (III) cannot intersect $H K, K M$ except at $M$, but $M$ is not on $H K, K O$, hence again $\Phi_{1}(u) \neq \Phi_{2}(v)$; for $2 \leqq u \leqq 3,2 \leqq$ $v<3$ the same conclusion follows from condition (IV); finally for $5 / 2 \leqq u \leqq 3, v=3, \Phi_{1}(u)$ is on the segment $O M$ while $\Phi_{2}(v)=L$, hence $\Phi_{1}(u) \neq \Phi_{2}(v)$ everywhere in the dotted region.

The diagonal $\Delta(t)=(t, t), 0 \leqq t \leqq 3$, is obviously homotopic to $\Sigma=A B C D E F G$ staying within the dotted region in which, as just proved, $\Xi(u, v)$ is never the origin. Hence

$$
\Psi(t)=\Phi_{1}(t)-\Phi_{2}(t)=(\Xi \circ \Delta)(t)
$$

is homotopic to $(E \circ \Sigma)(t)$ never hitting the origin, and therefore the winding numbers about the origin are the same.

It only remains to check that $\Xi \circ \Sigma$ has winding number -1 about the origin, which follows just by adding the winding numbers of $\Xi \circ A B, \Xi \circ B C, \Xi \circ C D, \Xi \circ D E, \Xi \circ E F, \Xi \circ F G$ which turn out to be respectively $0,-1 / 2$ (because of condition (II)), $0,0,-1 / 2$ (because of condition (II)), 0 .

Lemma 2. Let $\Phi_{1}$ and $\Phi_{2}$ be defined as above. Let $T$ be a homeomorphism defined on $\Phi_{1}$ as well as in its interior and such that $T\left(\Phi_{1}(t)\right)=\Phi_{2}(t), 0 \leqq t \leqq 3$. Then $T$ has a fixed point which is contained in the intersection of the interiors of $\Phi_{1}$ and $\Phi_{2}$.

Proof. Assume $T$ has no fixed point in the intersection of the interiors of the simple closed curves $\Phi_{1}, \Phi_{2}$. Then 


$$
H_{s}(t)=s \Phi_{1}(t)-T\left(s \Phi_{1}(t)\right)
$$

is a homotopy from the constant $-T(0)$ map to $\Phi_{1}(t)-\Phi_{2}(t)$ which never hits the origin. This contradicts Lemma 1.

THEOREM. Let $T$ be an orientation preserving homeomorphism defined in a subset of the plane and interchanging two points $P, Q$. Let $\Gamma$ be a simple curve joining $P$ to $Q$, and $\Omega$ a simply connected set contained in the domain of $T$ as well as in its range and such that $\Gamma \subset \Omega, T(\Gamma) \subset \Omega, T^{-1}(\Gamma) \subset \Omega$. Then $T$ has a fixed point in $\Omega$.

Proof. We show that the situation of Lemma 2 must occur. We may assume that the plane is coordinatized so that: $P=(1,0)$, $Q=(0,0)$, and $\Gamma$ is the segment $P Q$. We may also assume (replacing $P, Q$ with another pair of points if necessary) that $T$ interchanges no pair of points of $\Gamma$ between $P$ and $Q$. Let $\phi$ be the parametrization of $\Gamma$ given by $\phi(t)=(1-t, 0), 0 \leqq t \leqq 1$. Define:

$$
t_{1}=\inf \left\{t: \phi[0, t] \cap T \phi[0, t] \neq \varnothing \quad \text { or } \quad \phi[0, t] \cap T^{-1} \phi[0, t] \neq \varnothing\right\}
$$

(the set is nonempty since it contains the number 1 ). It is clear that either $\phi\left(t_{1}\right) \in T \phi\left[0, t_{1}\right]$ or $\phi\left(t_{1}\right) \in T^{-1} \phi\left[0, t_{1}\right]$. First, we will assume only one of these events occurs. Later, we will consider the case when both inclusions are valid.

By replacing $T$ by $T^{-1}$ if necessary, we may assume the second of the two inclusions, that is $\phi\left(t_{1}\right) \in T^{-1} \phi\left[0, t_{1}\right]$, or equivalently $T \phi\left(t_{1}\right) \in$ $\phi\left[0, t_{1}\right]$. Let $M=\phi\left(t_{1}\right), H=T^{-1}(M), L=T(M)$, and define $t_{0} \leqq t_{1}$ to be the scalar such that $\phi\left(t_{0}\right)=L$. We may assume $t_{0}<t_{1}$, otherwise $\phi\left(t_{1}\right)$ is a fixed point. Define $t_{-1}=\sup \left\{t \leqq t_{0}: T^{-1} \phi(t) \in T \phi\left[0, t_{1}\right]\right\}$, and let $N=T^{-1} \phi\left(t_{-1}\right)$. Finally, choose $t_{*}$ so that $T \phi\left(t_{*}\right)=N$ and let $K=$ $\phi\left(t_{*}\right)$. The situation is summarized in Figure 3. Now the three paths

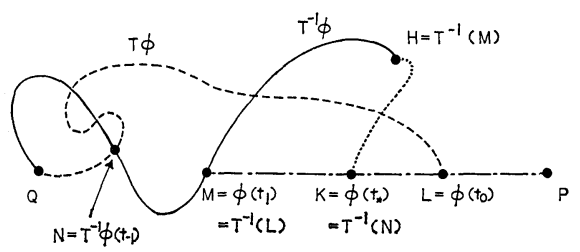

FIGURE 3

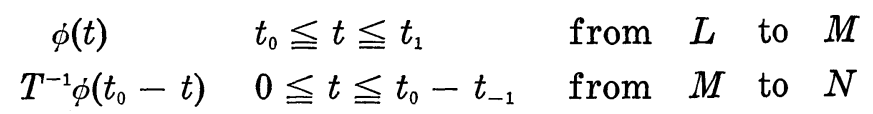

and

$T \phi(t) \quad t_{*} \leqq t \leqq t_{0} \quad$ from $N$ to $L$


form a simple closed curve which turns out to be contained in $\Omega$ (because it is composed by portions of $\Gamma, T(\Gamma), T^{-1}(\Gamma)$ ) together with its interior (because $\Omega$ is simply connected). Let $\Phi_{2}:[0,3] \rightarrow \mathscr{R}^{2}$ be a parametrization of this curve so that $\Phi_{2}[0,1]$ is the path $L M$, $\Phi_{2}[1,2]$ is the path $M N$, and $\Phi_{2}[2,3]$ is the path $N L$. Define $\Phi_{1}(t)=$ $T^{-1} \Phi_{2}(t)$ for $0 \leqq t \leqq 3$; notice that $\Phi_{1}[0,1]=T^{-1} \phi\left[t_{0}, t_{1}\right]$ is a path from $M$ to $H, \Phi_{1}[1,2]$ is a path (not necessarily in $\Omega$ ) from $H$ to $K$, and $\Phi_{1}[2,3]=\phi\left[t_{*}, t_{1}\right]$ is a path from $K$ to $M$. Since $T$ is a homeomorphism, $\Phi_{1}$ is also a simple closed curve, and by applyidg the Schoenflies Theorem and introducing a new coordinate system on the plane (which may have the opposite orientation of the old one) we may assume that $\Phi_{1}$ is identical to the triangle defined before Lemma 1 and that $\Phi_{2}$ satisfies condition (I) for an appropriate choice of $\lambda>0$. It only remains to show that $\Phi_{2}$ satisfies conditions (II), (III), and (IV). Since $T \Phi_{1}=\Phi_{2}$ and $T$ preserves orientation, condition (II) is immediate. Condition (IV) follows from the choice of $t_{1}$. It is easily seen that the set $C_{M N}=\Phi_{2}(1,2]$, which is the path from $M$ to $N$, is disjoint from $\Phi_{1}[2,3]$, so to verify condition (III) it suffices to show $C_{M N}$ is disjoint from $C_{H K}=\Phi_{1}(1,2]=\mathrm{T}^{-1} C_{M N}$, which is the path from $H$ to $K$. To do this, observe that the path $\phi\left[t_{-1}, t_{1}\right]$ followed by the path $C_{M N}$ is a simple curve, hence its image under $T^{-1}, C_{N M H K}$ is also free of self-intersections. But the sets $C_{M N}$ and $C_{H K}$ are disjoint portions of the set $C_{N M H K}$.

We return now to the case that both $\phi\left(t_{1}\right) \in T \phi\left[0, t_{1}\right]$ and $\phi\left(t_{1}\right) \epsilon$ $T^{-1} \phi\left[0, t_{1}\right]$. In this case we refer to Figure 4 . Let $M=\phi\left(t_{1}\right)$ and define $t_{0}$ and $t_{*}$ so that $M=T^{-1} \phi\left(t_{0}\right)=T \phi\left(t_{*}\right)$. We have $t_{*} \neq t_{0}$, since equality would violate the assumption made in the third sentence of this proof. Replacing $T$ by $T^{-1}$ if necessary, we may assume $t_{*}<t_{0}$. Let $H=\phi\left(t_{*}\right), K=\phi\left(t_{0}\right)$ and $N=T(K)$. The two paths

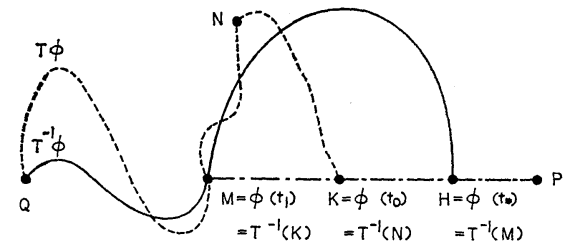

FIGURE 4

$$
\phi(t) t_{0} \leqq t \leqq t_{1} \text { from } K \text { to } M
$$

and

$$
T \phi(t) t_{*} \leqq t \leqq t_{1} \text { from } M \text { through } N \text { to } K
$$

form a simple closed curve; let $\Phi_{2}:[0,3] \rightarrow \mathscr{R}^{2}$ be a parametrization of this curve so that $\Phi_{2}[0,1]$ is the path $K M, \Phi_{2}[1,2]$ is the path 
$M N$, and $\Phi_{2}[2,3]$ is the path $N K$. Define $\Phi_{1}(t)=T^{-1} \Phi_{2}(t), 0 \leqq t \leqq 3$. The remainder of the proof in this case is analogous to the proof of the first case.

2. Remarks and examples. The proof of the theorem would become trivial if the hypotheses guaranteed the existence of a closed curve from $P$ through $Q$ to $P$ which is transformed into itself. But this is not always true, for if $T_{1}: \mathscr{R}^{2} \rightarrow \mathscr{R}^{2}$ is defined by $T_{1}(x, y)=$ $(-x,-y+\sin x)$ then $T_{1}$ is an orientation-preserving homeomorphism on $\mathscr{R}^{2}$ which interchanges the points $P=(\pi, 0)$ and $Q=(-\pi, 0)$; however, there exists no bounded connected set containing $P$ and $Q$ which is transformed into itself. It is interesting to note that this implies that $T_{1}$ is not an element of any flow on $\mathscr{R}^{2}$.

To see that the orientation-preserving hypothesis is necessary, consider the homeomorphism $T_{2}: \mathscr{R}^{2} \rightarrow \mathscr{R}^{2}$ defined by

$$
T_{2}(x, y)=\left\{\begin{array}{lll}
(-x, y) & \text { if } & |x| \geqq \frac{\pi}{2} \\
(-x, y+\cos x) & \text { if } & |x|<\frac{\pi}{2}
\end{array}\right.
$$

$T_{2}$ interchanges every pair of points $\{(x, y),(-x, y)\}$ for which $|x| \geqq$ $\pi / 2$, but has no fixed point.

We conclude with a simple corollary to the Theorem (with $\Omega=$ $\mathscr{R}^{2}$ ). Our notation follows that of [3].

CoRollary. Let $T: S^{2} \rightarrow S^{2}$ be a homeomorphism such that $T$ is of Brouwer degree 1, and $T$ interchanges two points. Then $T$ has two fixed points.

Proof. Standard results in algebraic topology (see [3] page 124, Exercise 3) show that $T$ has at least one fixed point, say $U$. Now $S^{2} \sim U$ is homeomorphic to $\mathscr{R}^{2}$; let $h: S^{2} \sim U \rightarrow \mathscr{R}^{2}$ be a homeomorphism. Then $h \circ T \circ h^{-1}$ is a homeomorphism of $\mathscr{R}^{2}$ which interchanges two points, and Theorem 34, page 122 of [3] shows that it also preserves orientation. Thus $h \circ T \circ h^{-1}$ has a fixed point, say $W$, and $V=h^{-1}(W)$ is another fixed point of $T$.

\section{REFERENCES}

1. S. A. Andrea, The plane is not compactly generated by a free mapping, Trans. Amer. Math. Soc., 151 (1970), 481-498.

2. L. E. J. Brouwer, Beweis des ebenen translationssatzes, Math. Ann., 72 (1912), 37-54. 3. John W. Keesee, An Introduction to Algebraic Topology, Brooks/Cole, Belmont, Calif. (1970).

Received July 31, 1974 and in revised form April 28, 1975. 


\section{PACIFIC JOURNAL OF MATHEMATICS}

\section{EDITORS}

RICHARD ARENS (Managing Editor) University of California

Los Angeles, California 90024

\section{R. A. Beaumont}

University of Washington Seattle, Washington 98105
J. DugundJI Department of Mathematics University of Southern Californıa Los Angeles, California 90007

D. Gilbarg aNd J. Milgram Stanford University Stanford, California 94305

\section{ASSOCIATE EDITORS}

E. F. BeCKENBACH

B. H. NeUmanN

F. Wolf

K. YoSHIDA

\section{SUPPORTING INSTITUTIONS}

UNIVERSITY OF BRITISH COLUMBIA CALIFORNIA INSTITUTE OF TECHNOLOGY UNIVERSITY OF CALIFORNIA MONTANA STATE UNIVERSITY UNIVERSITY OF NEVADA NEW MEXICO STATE UNIVERSITY OREGON STATE UNIVERSITY UNIVERSITY OF OREGON OSAKA UNIVERSITY
UNIVERSITY OF SOUTHERN CALIFORNIA STANFORD UNIVERSITY UNIVERSITY OF TOKYO UNIVERSITY OF UTAH WASHINGTON STATE UNIVERSITY UNIVERSITY OF WASHINGTON AMERICAN MATHEMATICAL SOCIETY NAVAL WEAPONS CENTER 


\section{Pacific Journal of Mathematics \\ Vol. 59, No. 1 \\ May, 1975}

Shashi Prabha Arya and M. K. Singal, More sum theorems for topological

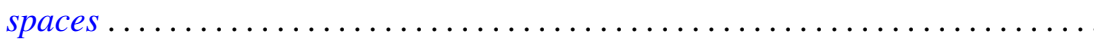

Goro Azumaya, F. Mbuntum and Kalathoor Varadarajan, On M-projective and

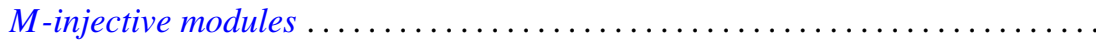

Kong Ming Chong, Spectral inequalities involving the infima and suprema of

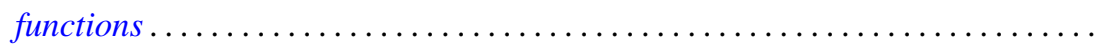

Alan Hetherington Durfee, The characteristic polynomial of the monodromy .......

Emilio Gagliardo and Clifford Alfons Kottman, Fixed points for orientation

preserving homeomorphisms of the plane which interchange two points ......

Raymond F. Gittings, Finite-to-one open maps of generalized metric spaces .......

Andrew M. W. Glass, W. Charles (Wilbur) Holland Jr. and Stephen H. McCleary,

$a^{*}$-closures of completely distributive lattice-ordered groups .............

Matthew Gould, Endomorphism and automorphism structure of direct squares of

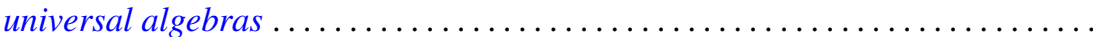

R. E. Harrell and Les Andrew Karlovitz, On tree structures in Banach spaces .....

Julien O. Hennefeld, Finding a maximal subalgebra on which the two Arens

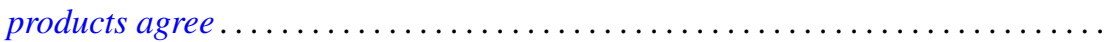

William Francis Keigher, Adjunctions and comonads in differential algebra .......

Robert Bernard Kelman, A Dirichlet-Jordan theorem for dual trigonometric

series

Allan Morton Krall, Stieltjes differential-boundary operators. III. Multivalued operators-linear relations...

Hui-Hsiung Kuo, On Gross differentiation on Banach spaces .

Tom Louton, A theorem on simultaneous observability ...

Kenneth Mandelberg, Amitsur cohomology for certain extensions of rings of algebraic integers.

Coy Lewis May, Automorphisms of compact Klein surfaces with boundary . . .

Peter A. McCoy, Generalized axisymmetric elliptic functions .

211

Muril Lynn Robertson, Concerning Siu's method for solving $y^{\prime}(t)=F(t$, $y(g(t)))$. .

Richard Lewis Roth, On restricting irreducible characters to normal

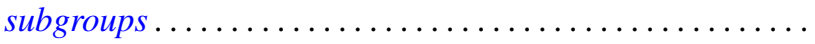

Albert Oscar Shar, $P$-primary decomposition of maps into an $H$-space .

Kenneth Barry Stolarsky, The sum of the distances to certain pointsets on the unit circle.

Bert Alan Taylor, Components of zero sets of analytic functions in $C^{2}$ in the unit ball or polydisc

Michel Valadier, Convex integrands on Souslin locally convex spaces ...

Januario Varela, Fields of automorphisms and derivations of $C$

Arnold Lewis Villone, A class of symmetric differential operators with deficiency

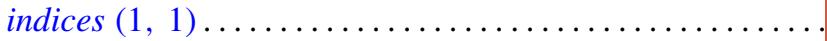

